\title{
Symétrie et reproductibilité temporelle des données neurographiques
}

$$
\text { Symmetry and temporal variability of neurography }
$$

\author{
O. Bouquiaux, A. Horward, F.C. Wang * \\ Département d'électroneuromyographie, service de médecine de l'appareil locomoteur, CHU Sart-Tilman, bâtiment B35, 4000 Liège, Belgique
}

Résumé

Ce travail a pour objectif de documenter les différences gauche/droite (G/D) et temporelle, entre 2 examens successifs (T1 et T2), des données neurographiques dans une population de 30 sujets volontaires sains (âge moyen : $22 \pm 2$ ans).

Méthodes. - Le protocole à T1 consiste à étudier bilatéralement : la conduction nerveuse motrice des nerfs médian, ulnaire, péronier et tibial avec mesure de la taille et de la latence de la réponse distale, latence et amplitude des ondes F et calcul de la vitesse de conduction (VC) motrice et de la persistance des ondes F ; la conduction nerveuse sensitive des nerfs médian, ulnaire, radial, cutané latéral et médial de l'avant-bras, sural et péronier superficiel avec mesure de la taille du potentiel sensitif et calcul de la VC sensitive. Le protocole à T2 (3 mois après T1) consiste à étudier les mêmes nerfs et paramètres du côté dominant.

Résultats et conclusion. - Une relation inverse est mise en évidence entre la variabilité des paramètres évaluant la VC nerveuse motrice et sensitive et la longueur du segment nerveux exploré. Les plus faibles différences G/D et temporelles sont donc mesurées pour la latence minimale des ondes $\mathrm{F}$ (entre 2 et $3 \%$ en moyenne). Les limites de symétrie et de variabilité temporelle sont particulièrement utiles, au diagnostic d'une neuropathie périphérique unilatérale ou au suivi neurophysiologique d'un patient, quand la variabilité du paramètre étudié est faible et lorsque la corrélation entre les valeurs enregistrées à gauche et à droite ou en T1 et T2 est élevée. Ceci se vérifie particulièrement pour l'amplitude motrice des nerfs ulnaire et tibial, l'amplitude sensitive du nerf radial et la latence minimale de l'onde F de tous les nerfs étudiés.

(C) 2003 Éditions scientifiques et médicales Elsevier SAS. Tous droits réservés.

\begin{abstract}
The aims of the present study are to document side-to-side differences and temporal variability, between two trials (T1 and T2 at a time interval of 3 months) of nerve conduction measurements collected from 30 healthy subjects (mean age $22 \pm 2$ years).

Methods. - The protocol at T1 consisted of motor nerve conduction studies of median, ulnar, peroneal and tibial nerves bilaterally, with measurement of (a) motor response size (amplitude and area); (b) terminal latency; (c) minimal, mean and maximal F-wave latency; (d) motor conduction velocity; and (e) F-wave occurrence. T1 also involved sensory nerve conduction studies of median, ulnar, radial, lateral and medial cutaneous, sural and superficial peroneal nerves bilaterally, with measurement of sensory potential size (amplitude and area) and computation of sensory conduction velocity. The protocol at T2 consisted of identical measurements from the dominant side.

Results and conclusion. - There was a negative relationship between the variability of parameters evaluating nervous conduction and the length of the nerve segment under study. Thus, the smallest side-to-side and temporal variabilities are measured for minimal F-wave latencies (on average 2-3\%). The limits of symmetry and temporal variability are particularly useful for diagnosis of unilateral peripheral neuropathy or neurophysiological follow-up of patients with neuropathy, when the variability of the parameter under study is weak and when there is a high correlation between values recorded on the left and on the right or at T1 and T2. This was the case for motor response size of tibial and ulnar nerves, sensory potential size of radial nerve and minimal F-wave latencies from each studied motor nerve.
\end{abstract}

(C) 2003 Éditions scientifiques et médicales Elsevier SAS. Tous droits réservés.

Mots clés : Valeurs normales ; Électrophysiologie ; Conduction nerveuse ; Neurographie

Keywords: Normal values; Electrophysiology; Nerve conduction studies; Neurography

* Auteur correspondant.

Adresse e-mail : fc.wang@chu.ulg.ac.be (F.C. Wang). 


\section{Introduction}

À ce jour, peu de travaux ont été consacrés à l'étude de la symétrie des données neurographiques chez le sujet sain [5]. Ce type d'étude a pourtant un intérêt potentiel dans l'établissement de limites de symétrie, permettant de considérer le patient examiné comme son propre témoin. Les valeurs normatives ne sont plus alors tributaires de certains paramètres que l'on sait influencer les données neurographiques tels que l'âge, la taille et la température cutanée (pour autant qu'elle soit similaire à gauche et à droite). Dans la pratique neurophysiologique de routine, il est habituel de considérer qu'une différence G/D de plus de $50 \%$ en amplitude $[14,19]$ et de plus de $15 \%$ en vitesse de conduction (VC) nerveuse constitue une différence significative. Un des buts assignés à ce travail est de vérifier si ces limites de symétrie quelque peu empiriques restent applicables en routine neurophysiologique et ce quel que soit le nerf moteur ou sensitif étudié.

Davantage d'études ont été consacrées à la reproductibilité des mesures de conduction nerveuse motrice ou sensitive chez des sujets sains et/ou chez des patients diabétiques $[1-3,6,11,12,16,18,23]$. La plupart de ces études se limitent souvent à établir le coefficient de variation entre plusieurs (2 ou davantage) séries de mesures réalisées à des temps distincts. En revanche, il est rarement fait mention dans ces mêmes travaux des valeurs normatives (limites supérieure et inférieure de la normale) de reproductibilité temporelle. Ces limites de reproductibilité temporelle sont pourtant cruciales lorsque l'on souhaite réaliser un suivi neurophysiologique de patients souffrant d'une neuropathie périphérique, en particulier dans le cadre des polyradiculonévrites chroniques (PRNC). Les limites de reproductibilité temporelle deviennent alors des limites de stabilité au-delà desquelles il est possible d'affirmer une aggravation ou une amélioration significative du paramètre étudié lors d'un contrôle neurophysiologique.

Dans ce travail, nous avons confronté la variabilité G/D à la variabilité temporelle des données neurographiques motrices et sensitives enregistrées dans une même population de 30 sujets volontaires sains.

\section{Matériel et méthodes}

\subsection{Population étudiée}

Nous avons examiné 30 sujets sains, volontaires ayant donné leur consentement éclairé, en respectant les critères d'inclusion suivants :

- absence d'antécédent neurologique, métabolique ou toxique ;

- absence de paresthésies ;

- absence d'anomalie à l'examen clinique comportant : une étude des réflexes ostéotendineux aux 4 membres, un testing musculaire analytique et une exploration de la sensibilité à la piqûre.

La population de référence comporte 15 hommes et 15 femmes dont l'âge moyen est de $22 \pm 2$ ans (valeurs extrêmes : 19-25) et dont la taille moyenne est de $174 \pm 10 \mathrm{~cm}$ (valeurs extrêmes : 158-188). Parmi les 30 sujets examinés, 25 sont droitiers et 5 gauchers.

En outre, l'utilité des valeurs normatives établies dans ce travail sera illustrée en confrontant les limites de reproductibilité temporelle aux suivis neurophysiologiques longitudinaux réalisés chez 2 patients dont l'un souffre d'un syndrome de Guillain-Barré et l'autre d'une polyradiculonévrite chronique (Fig. 4).

\subsection{Description des techniques neurographiques}

De très nombreuses techniques neurographiques sont décrites dans la littérature. Dans ce travail, le choix s'est porté sur des méthodes faisant partie de notre routine quotidienne depuis des années. En effet, cette étude n'a d'intérêt que si elle est réalisée par une personne rompue aux techniques et aux paramètres analysés introduisant une variabilité liée à l'expérimentateur la plus faible possible.

La neurographie motrice a porté sur les nerfs médian, ulnaire, péronier et tibial. L'électrode détectrice active (G1) est d'abord mise en place au point moteur du muscle étudié (court extenseur des orteils pour le nerf péronier, abducteur du gros orteil pour le nerf tibial, court abducteur du pouce pour le nerf médian et abducteur du petit doigt pour le nerf ulnaire). La distance entre la cathode lors de la stimulation distale et $\mathrm{G} 1$ est de $8 \mathrm{~cm}$ pour tous les nerfs étudiés. Les distances sont mesurées à l'aide d'un mètre ruban soit d'un point de stimulation (centre de la cathode) proximal à un point de stimulation distal pour le calcul des VC, soit du point de stimulation distal (centre de la cathode) au centre de G1 pour l'enregistrement des réponses distales motrices. Pour tous les nerfs à l'exception du nerf médian, les distances sont mesurées en ligne droite. La distance distale du nerf médian $(8 \mathrm{~cm})$ est mesurée en tentant de respecter le trajet anatomique du nerf (de G1 au milieu du premier pli palmaire du poignet avec ensuite modification de direction pour être dans l'axe de l'avant-bras). Les réponses motrices distales sont analysées en termes d'amplitude (de la ligne de base au sommet du premier pic négatif), de surface (surface du pic négatif initial) et de latence (temps écoulé entre la stimulation et le début de la réponse motrice). La VC motrice est calculée uniquement pour le nerf péronier au niveau du segment jambier et lors de la traversée du genou. Pour chacun des nerfs étudiés, des réponses $F$ sont évoquées par des stimulations supramaximales appliquées à la cheville ou au poignet. Au minimum 20 stimulations sont délivrées pour les nerfs médian, ulnaire et péronier, tandis que le nombre de stimulations est limité à 10 pour le nerf tibial. Concernant les réponses $\mathrm{F}$, les paramètres suivants sont analysés : latence (latence de la réponse $\mathrm{F}-$ la latence distale motrice) minimale, moyenne et maximale, persistance (pourcentage de réponses $\mathrm{F}$ enregistrées par rapport au nombre de stimulations délivrées) et amplitude maximale pic à pic après superposition de l'ensemble des tracés (Fig. 1).

Aux membres supérieurs, les nerfs sensitifs suivants sont étudiés : médian, ulnaire, radial, cutanés médial et latéral de 


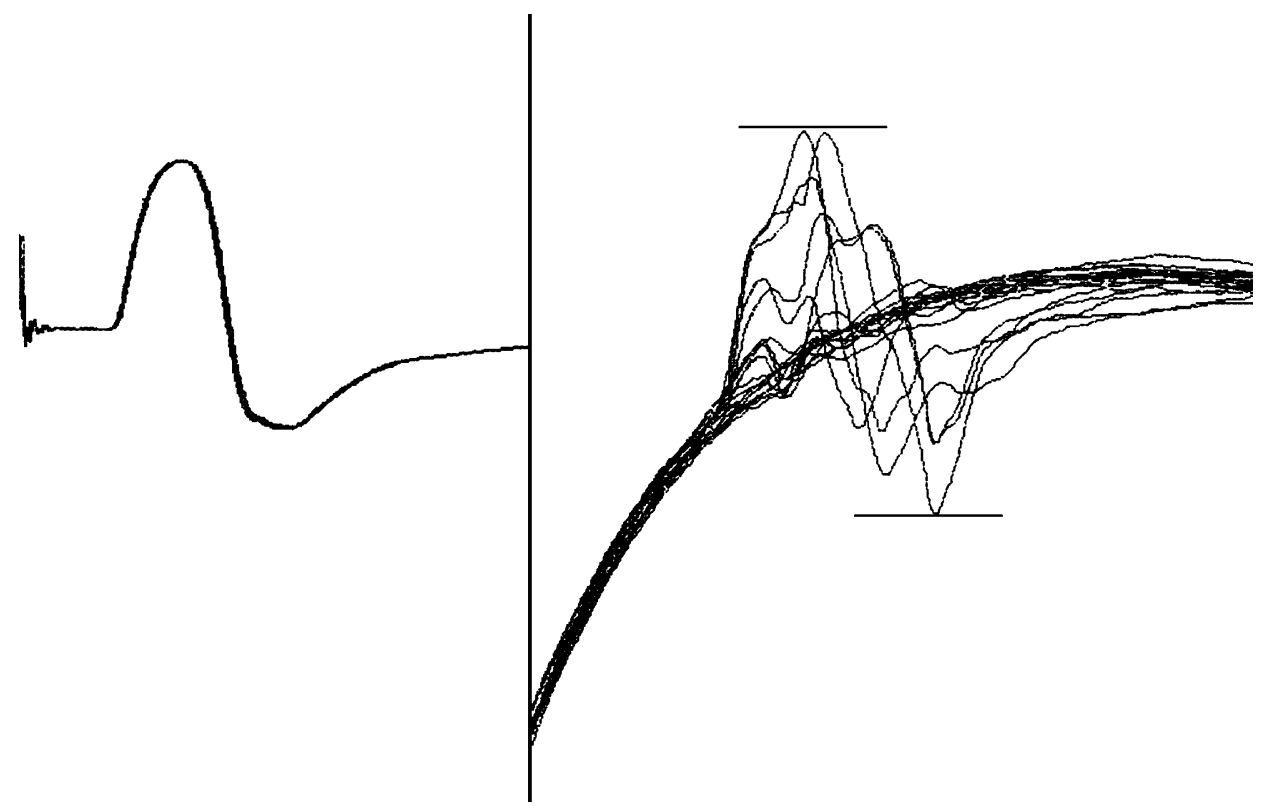

Fig. 1. Mesure le l'amplitude de l'onde F pic à pic (traits horizontaux) après superposition des différentes traces.

l'avant-bras. Aux membres inférieurs, les potentiels sensitifs des nerfs sural et péronier superficiel sont enregistrés. Les potentiels sensitifs obtenus sont analysés en termes d'amplitude et de surface et la VC sensitive est calculée. Le logiciel utilisé offre le choix de 4 «algorithmes » pour la mesure d'amplitude et de 3 pour la mesure de la surface. Nous avons choisi de mesurer la surface délimitée par le pic négatif initial et la droite reliant le sommet du premier pic positif (ou la ligne de base en l'absence de pic positif initial) au sommet du second pic positif (ou du premier pic positif en l'absence de pic positif initial). L'amplitude est donnée par la verticale passant par le sommet du pic négatif initial et la base de la surface prédéfinie (Fig. 2). Pour les nerfs médian et ulnaire, la conduction orthodromique est évaluée par une stimulation palmaire percutanée. La distance entre le centre de la cathode et le milieu de G1 (situé environ 1 à $2 \mathrm{~cm}$ proximalement par

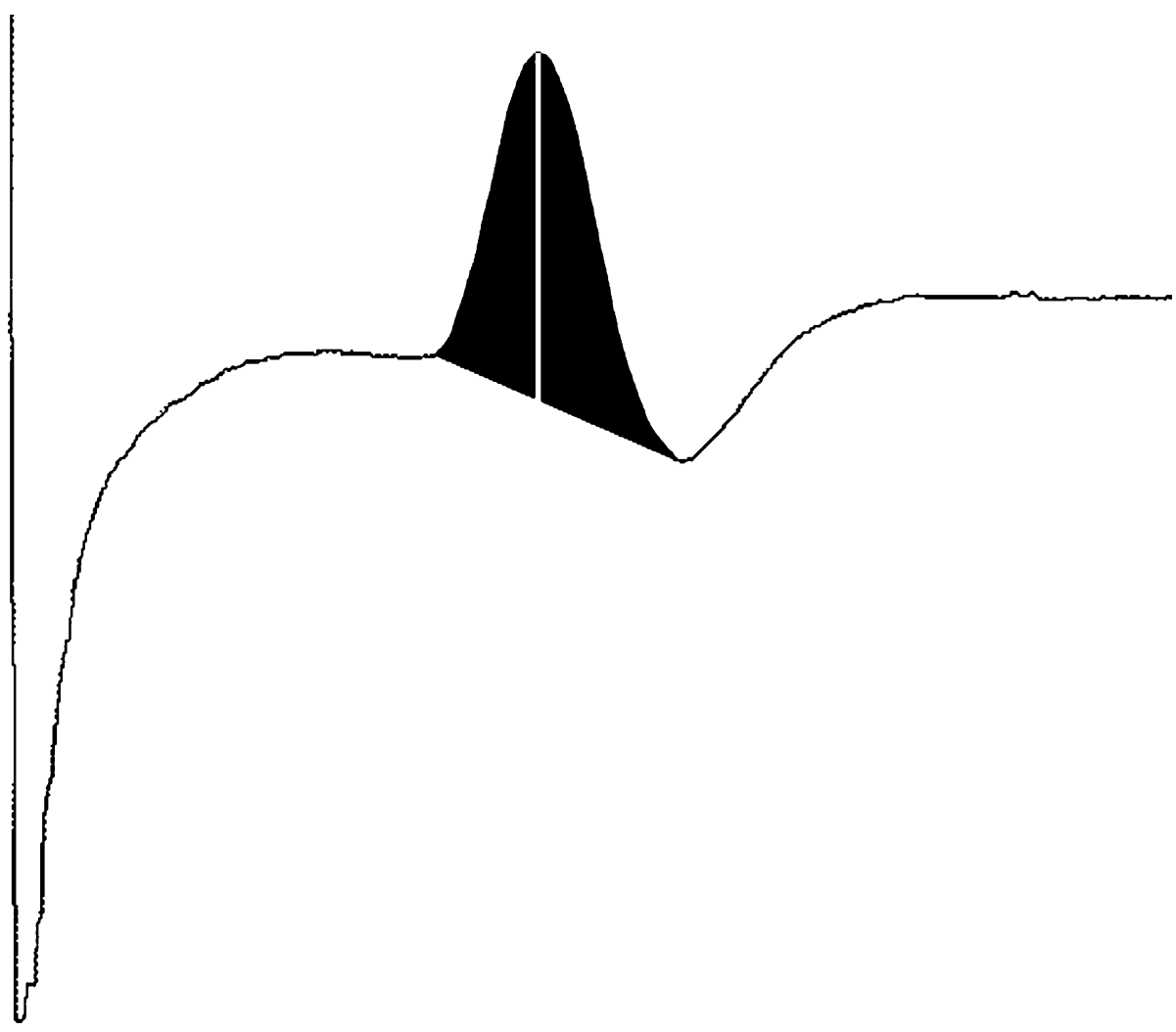

Fig. 2. Mesure de l'amplitude (trait vertical blanc) et de la surface (aire en noir) des potentiels sensitifs. 
rapport au premier pli palmaire du poignet) est de 8 ou $9 \mathrm{~cm}$ en fonction de la longueur de la main du sujet examiné. Pour tous les autres nerfs sensitifs, la conduction antidromique est étudiée, avec une distance entre le centre de la cathode et le milieu de G1 de $14 \mathrm{~cm}$ pour les nerfs cutané latéral de l'avant-bras (du pli du coude, latéralement par rapport au tendon inférieur du biceps, au point situé $14 \mathrm{~cm}$ distalement sur une ligne reliant le tendon inférieur du biceps au pouls radial) et sural (de la région rétromalléolaire externe au mollet), de $12 \mathrm{~cm}$ pour les nerfs cutané médial de l'avant-bras (à la face interne du membre supérieur, $4 \mathrm{~cm}$ au-dessus et $8 \mathrm{~cm}$ en dessous du pli du coude) et radial (de l'angle formé par les 2 premiers métacarpiens au bord externe de l'avantbras), et de $8 \mathrm{~cm}$ pour le nerf péronier superficiel (de la région directement sus-malléolaire externe, ou en situation quelque peu plus antérieure, à la face latérale de la jambe).

Toutes les données neurographiques motrices et sensitives sont enregistrées par un même électromyographe de marque Medtronic disposant du logiciel Keypoint dans sa version 2.12. La stimulation nerveuse électrique bipolaire est délivrée par un stimulateur de la firme Medelec (Modèle 16893T, distance interélectrode $=2,5 \mathrm{~cm}$ ). Les potentiels moteurs et sensitifs sont évoqués par des stimulations électriques de courant constant et rectangulaire. Les nerfs moteurs sont activés par des impulsions de $0,2 \mathrm{~ms}$ de durée à la fréquence de $1 \mathrm{~Hz}$; tandis que les nerfs sensitifs sont stimulés par des chocs de $0,1 \mathrm{~ms}$ de durée à la fréquence de $3 \mathrm{~Hz}$. Les enregistrements de surface des réponses motrices sont assurés par des électrodes disposables $\mathrm{Ag} / \mathrm{AgCl}$ de marque Nicolet (surface de contact $=20 \times 25 \mathrm{~mm}$ ). L'électrode active est placée au plus près de la zone des plaques motrices. L'électrode de référence est fixée au dos de la première phalange du pouce (nerf médian) et de l'auriculaire (nerf ulnaire) ou à la base du premier orteil (nerf tibial) et du cinquième orteil (nerf péronier). Les enregistrements de surface des réponses sensitives sont effectués par une paire d'électrodes ( 2 feutres dont la surface d'enregistrement $=20 \times 7 \mathrm{~mm}$ assurent le contact) enchâssées dans une structure plastique (distance interélectrode $=1,8 \mathrm{~cm}$ ) et fabriquées par la firme Medelec (modèle 16933). Les curseurs de latence et d'amplitude sont positionnés automatiquement sans correction manuelle.

Dans un premier temps (T1), les différentes techniques neurographiques motrices et sensitives sont appliquées bilatéralement chez les 30 sujets sains et les différences G/D sont analysées. Un second examen (T2) est réalisé à 3 mois d'intervalle de façon unilatérale (côté dominant) chez les mêmes 30 sujets volontaires et les différences temporelles sont analysées. Pour étudier l'influence de la température sur la variabilité temporelle, nous avons mesuré la température cutanée à l'emplacement de l'électrode détectrice active (G1) pour chacune des mesures sensitives et motrices réalisées. En effet, il a été démontré que la corrélation entre la température et la taille des réponses neurographiques augmente plus la zone de mesure de la température cutanée est proche du site de détection des potentiels évoqués $[3,17]$. Toutes les données sont recueillies à température ambiante sans réchauffement du membre étudié et sans correction de la valeur mesurée si la température cutanée s'avère basse. Enfin, sachant que les paramètres d'amplitude et de surface des potentiels moteurs et sensitifs seraient sujets à une grande variabilité et que cette variabilité serait en grande partie tributaire de modifications de position d'électrode détectrice $[4,20]$, nous avons choisi d'optimiser ces paramètres à T2 . Autrement dit, chaque fois que les paramètres d'amplitude ou de surface à T2 étaient supérieurs ou égaux à ceux enregistrés à T1, les valeurs obtenues étaient conservées telles quelles. En revanche, lorsque la taille des réponses motrices ou sensitives à $\mathrm{T} 2$ était inférieure à celle enregistrée à $\mathrm{T} 1$, la position des électrodes détectrices était modifiée pour tenter d'obtenir une réponse de plus grande taille. Dans l'exposé des résultats et leur discussion, seules les valeurs d'amplitude et de surface à $\mathrm{T} 2$ après optimisation sont prises en compte.

\subsection{Analyse statistique}

Les différences G/D et temporelles sont analysées sur le plan statistique en termes de coefficient de corrélation, de différences absolue et pourcentuelle ainsi qu'en terme de coefficient de variation (CV). Le coefficient $r$ est calculé dans l'analyse de corrélation de type Bravais-Pearson. Les différences absolues sont obtenues en soustrayant les valeurs enregistrées soit à gauche et à droite, soit en T1 et en T2, et en considérant la différence en faisant abstraction du signe de celle-ci. Les différences pourcentuelles se calculent en divisant la différence absolue par la moyenne $(m)$ des valeurs enregistrées à gauche et à droite ou en T1 et T2 $\left(m_{1}\right.$ et $\left.m_{2}\right)$. Les limites de symétrie G/D et de variabilité temporelle sont établies par la méthode du percentile en fixant la limite au P95. La méthode du percentile offre l'avantage d'être applicable à des distributions non-gaussiènes qui caractérisent certains paramètres neurographiques, en particulier les amplitudes et surfaces des réponses sensitives et motrices. Le coefficient de variation apprécie en pourcentage la reproductibilité ou encore la variabilité analytique des mesures enregistrées chez les 30 sujets sains $(n=30)$ lors de 2 tests successifs $\left(x_{1}\right.$ et $\left.x_{2}\right)$. Il se calcule par la formule :

$$
\mathrm{CV}=(\mathrm{DS} / m) \times 100 \%
$$

où

DS $($ écart - type $)=\sqrt{\Sigma\left(X_{1}-X_{2}\right)^{2} / 2 n}$ et $\mathrm{m}=\left(m_{1}+m_{2}\right) / 2$.

L'influence de la température et de l'intensité de stimulation sur la variabilité temporelle est analysée par une étude de covariance.

\section{Résultats}

L'ensemble des résultats concernant les différences G/D et temporelles ainsi que les limites des valeurs normatives (P95) est présenté dans les Tableaux 1-3.

\subsection{Variabilités gauche/droite}

Les variabilités G/D les plus faibles concernent les paramètres évaluant la $\mathrm{VC}$ nerveuse ; viennent ensuite la taille des réponses motrices et sensitives, et enfin les paramètres d'amplitude et de persistance des réponses $\mathrm{F}$ (Tableaux 1 et 2). 
Tableau 1

Résultats de la neurographie motrice chez 30 sujets volontaires sains

\begin{tabular}{|c|c|c|c|c|c|}
\hline & & Ulnaire & Médian & Péronier & Tibial \\
\hline Amplitude & $m$ (DS) & $11,7(2,2)$ & $9,5(1,3)$ & $7,8(1,6)$ & $13,9(3,5)$ \\
\hline \multirow[t]{2}{*}{$(\mathrm{mV})$} & $\Delta \% \mathrm{G} / \mathrm{D}$ & 10 & 11 & 14 & 14 \\
\hline & $\Delta \% \mathrm{~T} 1 / \mathrm{T} 2$ & 6 & 9 & 10 & 8 \\
\hline Surface & $m(\mathrm{DS})$ & $32,3(6,5)$ & $30,6(6,8)$ & $24,7(5,6)$ & $36,0(9,3)$ \\
\hline \multirow[t]{2}{*}{ (mV.ms) } & $\Delta \% \mathrm{G} / \mathrm{D}$ & 9 & 13 & 17 & 13 \\
\hline & $\Delta \% \mathrm{~T} 1 / \mathrm{T} 2$ & 9 & 13 & 16 & 13 \\
\hline VCM jambe & $m(\mathrm{DS})$ & & & $48,4(4,1)$ & \\
\hline \multirow[t]{2}{*}{$(\mathrm{m} / \mathrm{s})$} & $\Delta \% \mathrm{G} / \mathrm{D}$ & & & 4 & \\
\hline & $\Delta \% \mathrm{~T} 1 / \mathrm{T} 2$ & & & 4 & \\
\hline \multirow{3}{*}{$\begin{array}{l}\text { VCM genou } \\
(\mathrm{m} / \mathrm{s})\end{array}$} & $m(\mathrm{DS})$ & & & $49,7(4,8)$ & \\
\hline & $\Delta \% \mathrm{G} / \mathrm{D}$ & & & 7 & \\
\hline & $\Delta \% \mathrm{~T} 1 / \mathrm{T} 2$ & & & 5 & \\
\hline LDM & $m(\mathrm{DS})$ & $2,8(0,3)$ & $3,4(0,3)$ & $3,9(0,4)$ & $4,1(0,3)$ \\
\hline \multirow[t]{2}{*}{$(\mathrm{ms})$} & $\Delta \% \mathrm{G} / \mathrm{D}$ & 7 & 6 & 6 & 7 \\
\hline & $\Delta \% \mathrm{~T} 1 / \mathrm{T} 2$ & 5 & 6 & 5 & 8 \\
\hline F-Lat minimale & $m(\mathrm{DS})$ & $23,6(1,9)$ & $22,4(1,8)$ & $42,2(3,8)$ & $43,2(3,9)$ \\
\hline \multirow[t]{2}{*}{$(\mathrm{ms})$} & $\Delta \% \mathrm{G} / \mathrm{D}$ & 3 & 3 & 3 & 2 \\
\hline & $\Delta \% \mathrm{~T} 1 / \mathrm{T} 2$ & 2 & 2 & 3 & 2 \\
\hline F-Lat moyenne & $m(\mathrm{DS})$ & $24,7(1,9)$ & $23,6(1,9)$ & $44,4(3,8)$ & \\
\hline \multirow[t]{2}{*}{ (ms) } & $\Delta \% \mathrm{G} / \mathrm{D}$ & 2 & 3 & 3 & \\
\hline & $\Delta \% \mathrm{~T} 1 / \mathrm{T} 2$ & 2 & 2 & 3 & \\
\hline F-Lat maximale & $m(\mathrm{DS})$ & $26,3(2,1)$ & $25,9(2,4)$ & $47,7(4,3)$ & \\
\hline \multirow[t]{2}{*}{ (ms) } & $\Delta \% \mathrm{G} / \mathrm{D}$ & 3 & 8 & 5 & \\
\hline & $\Delta \% \mathrm{~T} 1 / \mathrm{T} 2$ & 3 & 7 & 5 & \\
\hline F-Amp & $m(\mathrm{DS})$ & $530(251)$ & $551(302)$ & 303 (179) & 746 (318) \\
\hline \multirow[t]{2}{*}{$(\mu \mathrm{V})$} & $\Delta \% \mathrm{G} / \mathrm{D}$ & 38 & 44 & 66 & 26 \\
\hline & $\Delta \% \mathrm{~T} 1 / \mathrm{T} 2$ & 38 & 40 & 46 & 34 \\
\hline F-Per & $m(\mathrm{DS})$ & $98(5)$ & $89(11)$ & $64(24)$ & $100(0)$ \\
\hline \multirow[t]{2}{*}{$(\%)$} & $\Delta \% \mathrm{G} / \mathrm{D}$ & 3 & 10 & 33 & 0 \\
\hline & $\Delta \% \mathrm{~T} 1 / \mathrm{T} 2$ & 3 & 10 & 35 & 0 \\
\hline
\end{tabular}

VCM, vitesse de conduction motrice ; LDM, latence distale motrice ; F-Lat, latence de l'onde F; F-Amp, amplitude de l'onde F; F-Per, persistance de l'onde $\mathrm{F} ; m$, moyenne ; DS, déviation standard ; $\Delta \% \mathrm{G} / \mathrm{D}$, variabilité gauche/droite pourcentuelle ; $\Delta \% \mathrm{~T} 1 / \mathrm{T} 2$, variabilité temporelle pourcentuelle.

Il existe une relation inverse entre l'importance de la variabilité $\mathrm{G} / \mathrm{D}$ des paramètres évaluant la $\mathrm{VC}$ nerveuse et la longueur du segment examiné. En effet, sur le plan moteur, la plus faible variabilité $\mathrm{G} / \mathrm{D}$ concerne la latence minimale de l'onde $\mathrm{F}$ qui explore le plus long segment du système nerveux périphérique. Viennent ensuite par ordre croissant de variabilité $\mathrm{G} / \mathrm{D}$, la $\mathrm{VC}$ motrice du péronier au niveau du segment jambier (environ $30 \mathrm{~cm}$ ), la VC motrice du péronier lors de la traversée du genou (environ $10 \mathrm{~cm}$ ) et les latences distales motrices $(8 \mathrm{~cm})$ (Tableau 1). Des résultats similaires sont obtenus concernant les VC sensitives. En effet, la variabilité $\mathrm{G} / \mathrm{D}$ est d'autant plus faible que la distance entre la cathode stimulatrice et G1 est importante (Tableau 2).

Les variabilités $G / D$ des latences moyennes et minimales de l'onde F sont comparables ; tandis que la latence maximale de l'onde $\mathrm{F}$ présente une variabilité plus importante (Tableau 1).

Tableau 2

Résultats de la neurographie sensitive chez 30 sujets volontaires sains

\begin{tabular}{lllllllll}
\hline & & Cutané médial & Ulnaire & Médian & Cutané latéral & Radial & $\begin{array}{l}\text { Péronier } \\
\text { superficiel }\end{array}$ \\
\hline Amplitude & $m(\mathrm{DS})$ & $16,4(4,3)$ & $32,9(11,7)$ & $59,0(17,7)$ & $18,6(6,2)$ & $35,6(10,1)$ & $32,4(10,9)$ & $23,0(7,3)$ \\
$(\mu \mathrm{V})$ & $\Delta \% \mathrm{G} / \mathrm{D}$ & 34 & 19 & 14 & 23 & 11 & 19 & 15 \\
& $\Delta \% \mathrm{~T} 1 / \mathrm{T} 2$ & 6 & 17 & 18 & 7 & 6 & 16 \\
Surface & $m(\mathrm{DS})$ & $6,1(1,6)$ & $14,1(4,9)$ & $24,9(7,6)$ & $7,8(2,4)$ & $15,5(4,3)$ & $16,5(5,2)$ & $13,5(4,4)$ \\
$(\mu \mathrm{V} . \mathrm{ms})$ & $\Delta \% \mathrm{G} / \mathrm{D}$ & 34 & 19 & 14 & 22 & 9 & 20 & 15 \\
& $\Delta \% \mathrm{~T} 1 / \mathrm{T} 2$ & 6 & 16 & 22 & 8 & 7 & 57 & 24 \\
$\mathrm{VCS}$ & $m(\mathrm{DS})$ & $65,6(3,8)$ & $65,3(4,3)$ & $61,9(4,1)$ & $63,2(3,4)$ & $62,3(3,9)$ & $57,7(3,5)$ & $54,8(4,0)$ \\
$(\mathrm{m} / \mathrm{s})$ & $\Delta \% \mathrm{G} / \mathrm{D}$ & 5 & 6 & 5 & 2 & 3 & 4 & 5 \\
& $\Delta \% \mathrm{~T} 1 / \mathrm{T} 2$ & 5 & 6 & 6 & 4 & 4 & 5 & 5 \\
\hline
\end{tabular}

VCS, vitesse de conduction sensitive ; $m$, moyenne ; DS, déviation standard ; $\Delta \% \mathrm{G} / \mathrm{D}$, variabilité gauche/ droite pourcentuelle) ; $\Delta \%$ T1/T2, variabilité temporelle pourcentuelle. 
Tableau 3

Coefficients de variation (CV) et de corrélation $(r)$ avec limites de variabilité absolues (P95) et pourcentuelles (P95 \%) des données neurographiques chez 30 sujets volontaires sains

\begin{tabular}{|c|c|c|c|c|c|c|c|c|}
\hline Paramètres & Nerfs & $\mathrm{CV}$ & $r(\mathrm{G} / \mathrm{D})$ & $r(\mathrm{~T} 1 / \mathrm{T} 2)$ & P95 (G/D) & P95 (T1/T2) & P95 \% (G/D) & $\mathrm{P} 95 \%(\mathrm{~T} 1 / \mathrm{T} 2)$ \\
\hline \multirow{7}{*}{$\begin{array}{l}\text { Amplitude } \\
\text { sensitive }\end{array}$} & Cutané médial & 6 & $<0,50$ & 0,94 & $14 \mu \mathrm{V}$ & $3 \mu \mathrm{V}$ & 69 & 21 \\
\hline & Ulnaire & 18 & 0,70 & 0,78 & $17 \mu \mathrm{V}$ & $19 \mu \mathrm{V}$ & 55 & 49 \\
\hline & Médian & 18 & 0,83 & 0,81 & $21 \mu \mathrm{V}$ & $24 \mu \mathrm{V}$ & 41 & 47 \\
\hline & Cutané latéral & 6 & 0,70 & 0,97 & $9 \mu \mathrm{V}$ & $3 \mu \mathrm{V}$ & 51 & 18 \\
\hline & Radial & 6 & 0,88 & 0,96 & $10 \mu \mathrm{V}$ & $6 \mu \mathrm{V}$ & 25 & 17 \\
\hline & $\begin{array}{l}\text { Péronier } \\
\text { superficiel }\end{array}$ & 15 & 0,63 & 0,82 & $18 \mu \mathrm{V}$ & $15 \mu \mathrm{V}$ & 62 & 41 \\
\hline & Sural & 16 & 0,80 & 0,76 & $10 \mu \mathrm{V}$ & $11 \mu \mathrm{V}$ & 33 & 44 \\
\hline \multirow{4}{*}{$\begin{array}{l}\text { Amplitude } \\
\text { motrice }\end{array}$} & Ulnaire & 6 & 0,77 & 0,91 & $2,7 \mathrm{mV}$ & $1,7 \mathrm{mV}$ & 25 & 23 \\
\hline & Médian & 9 & 0,67 & 0,70 & $2,2 \mathrm{mV}$ & $3,2 \mathrm{mV}$ & 29 & 29 \\
\hline & Péronier & 9 & 0,72 & 0,89 & $2,6 \mathrm{mV}$ & $2,1 \mathrm{mV}$ & 41 & 31 \\
\hline & Tibial & 7 & 0,76 & 0,93 & $4,5 \mathrm{mV}$ & $2,3 \mathrm{mV}$ & 34 & 19 \\
\hline \multirow[t]{7}{*}{ VCS } & Cutané médial & 4 & 0,53 & 0,55 & $7,8 \mathrm{~ms}$ & $6,4 \mathrm{~ms}$ & 12 & 9 \\
\hline & Ulnaire & 5 & 0,78 & 0,64 & $8,4 \mathrm{~ms}$ & $11,3 \mathrm{~ms}$ & 15 & 18 \\
\hline & Médian & 5 & 0,70 & 0,46 & $7,1 \mathrm{~ms}$ & $7,2 \mathrm{~ms}$ & 11 & 12 \\
\hline & Cutané latéral & 4 & 0,83 & 0,61 & $3,3 \mathrm{~ms}$ & $5,3 \mathrm{~ms}$ & 5 & 9 \\
\hline & Radial & 3 & 0,86 & 0,73 & $3,7 \mathrm{~ms}$ & $5,5 \mathrm{~ms}$ & 6 & 9 \\
\hline & $\begin{array}{l}\text { Péronier } \\
\text { superficiel }\end{array}$ & 5 & 0,62 & 0,55 & $9,4 \mathrm{~ms}$ & $9,8 \mathrm{~ms}$ & 14 & 16 \\
\hline & Sural & 4 & 0,74 & 0,69 & $5,5 \mathrm{~ms}$ & $5,8 \mathrm{~ms}$ & 11 & 11 \\
\hline \multirow[t]{2}{*}{ VCM } & $\begin{array}{l}\text { Péronier } \\
\text { (jambe) }\end{array}$ & 3 & 0,71 & 0,73 & $4,4 \mathrm{~m} / \mathrm{s}$ & $5,2 \mathrm{~m} / \mathrm{s}$ & 10 & 11 \\
\hline & $\begin{array}{l}\text { Péronier } \\
\text { (genou) }\end{array}$ & 5 & 0,51 & 0,74 & $8,2 \mathrm{~m} / \mathrm{s}$ & $5,6 \mathrm{~m} / \mathrm{s}$ & 16 & 12 \\
\hline \multirow[t]{4}{*}{ LDM } & Ulnaire & 6 & 0,76 & 0,79 & $0,5 \mathrm{~ms}$ & $0,5 \mathrm{~ms}$ & 15 & 15 \\
\hline & Médian & 5 & 0,67 & 0,62 & $0,5 \mathrm{~ms}$ & $0,3 \mathrm{~ms}$ & 14 & 13 \\
\hline & Péronier & 5 & 0,78 & 0,79 & $0,5 \mathrm{~ms}$ & $0,5 \mathrm{~ms}$ & 12 & 12 \\
\hline & Tibial & 8 & 0,46 & 0,43 & $1,3 \mathrm{~ms}$ & $1,0 \mathrm{~ms}$ & 29 & 23 \\
\hline \multirow[t]{4}{*}{ F-Lat minimale } & Ulnaire & 2 & 0,93 & 0,95 & $1,4 \mathrm{~ms}$ & $1,1 \mathrm{~ms}$ & 6 & 5 \\
\hline & Médian & 2 & 0,94 & 0,95 & $1,3 \mathrm{~ms}$ & $1,0 \mathrm{~ms}$ & 6 & 5 \\
\hline & Péronier & 4 & 0,96 & 0,88 & $3,5 \mathrm{~ms}$ & $3,7 \mathrm{~ms}$ & 7 & 9 \\
\hline & Tibial & 2 & 0,94 & 0,93 & $3,0 \mathrm{~ms}$ & $3,5 \mathrm{~ms}$ & 7 & 8 \\
\hline \multirow[t]{4}{*}{ F-Amp } & Ulnaire & 35 & 0,64 & 0,50 & $380 \mu \mathrm{V}$ & $554 \mu \mathrm{V}$ & 82 & 100 \\
\hline & Médian & 38 & $<0,50$ & $<0,50$ & $692 \mu \mathrm{V}$ & $478 \mu \mathrm{V}$ & 111 & 112 \\
\hline & Péronier & 31 & $<0,50$ & 0,71 & $374 \mu \mathrm{V}$ & $257 \mu \mathrm{V}$ & 128 & 135 \\
\hline & Tibial & 34 & $<0,50$ & $<0,50$ & $471 \mu \mathrm{V}$ & $610 \mu \mathrm{V}$ & 76 & 96 \\
\hline \multirow[t]{4}{*}{ F-Per } & Ulnaire & 4 & $<0,50$ & $<0,50$ & $10 \%$ & $15 \%$ & 11 & 16 \\
\hline & Médian & 10 & $<0,50$ & $<0,50$ & $25 \%$ & $20 \%$ & 33 & 29 \\
\hline & Péronier & 26 & 0,86 & 0,66 & $30 \%$ & $50 \%$ & 100 & 133 \\
\hline & Tibial & 0 & 1 & 1 & $0 \%$ & $0 \%$ & 0 & 0 \\
\hline
\end{tabular}

VCS, vitesse de conduction sensitive ; VCM, vitesse de conduction motrice ; LDM, latence distale motrice ; F-Lat, latence de l'onde F; F-Amp, amplitude de l'onde F ; F-Per, persistance de l'onde F.

Concernant la taille des réponses motrices et sensitives, les variabilités $\mathrm{G} / \mathrm{D}$ sont globalement identiques que la taille des réponses soit évaluée par l'amplitude ou la surface du pic négatif initial (Tableaux 1 et 2).

La variabilité $\mathrm{G} / \mathrm{D}$ du paramètre persistance des réponses F est inversement proportionnelle $(r=0,999)$ à la persistance moyenne des réponses $\mathrm{F}$ selon le nerf étudié (Fig. 3A). Autrement dit, pour un nerf donné, plus la persistance est élevée et plus la variabilité G/D est faible.

L'étude de corrélation entre les valeurs enregistrées à gauche et à droite révèle un coefficient $r$ élevé $(>0,75)$ particulièrement pour les paramètres et nerfs suivants (Tableau 3) :
- la latence minimale de l'onde F pour tous les nerfs étudiés ;

- les VC sensitives des nerfs ulnaire, cutané latéral de l'avant-bras et radial ;

- les latences distales motrices des nerfs ulnaire et péronier;

- l'amplitude du potentiel sensitif du nerf radial ;

- l'amplitude du potentiel moteur des nerfs ulnaire et tibial.

\subsection{Variabilités $T 1 / T 2$}

Globalement, les différences T1/T2 concernant les différents nerfs et paramètres étudiés sont similaires aux différen- 


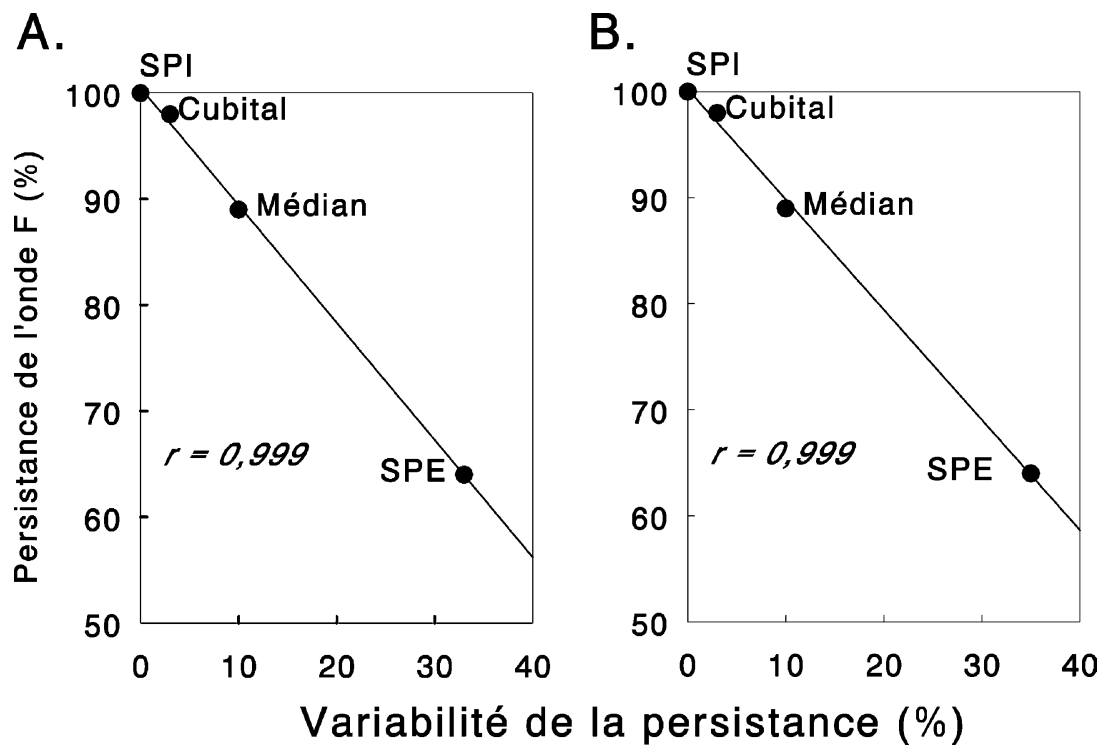

Fig. 3. Corrélations entre les valeurs moyennes de la persistance de l'onde F et des variabilités gauche/droite (A) et temporelle (B) de la persistance, établies dans un groupe de 30 sujets sains.

ces G/D (Tableaux 1 et 2). En effet, ici aussi les variabilités les plus faibles concernent les paramètres évaluant la VC nerveuse motrice ou sensitive. Une relation inverse entre l'importance de la variabilité de ces paramètres et la longueur du segment examiné est retrouvée. Les variabilités les plus élevées concernent les paramètres d'amplitude et de persistance des réponses F. La variabilité temporelle relative à la taille des réponses motrices et sensitives se situe entre ces 2 extrêmes. Une relation inverse $(r=0,999)$ entre la variabilité de la persistance de l'onde $\mathrm{F}$ et la persistance moyenne, en fonction du nerf étudié, est également constatée (Fig. 3B).

L'amplitude sensitive des nerfs cutanés latéral et médial de l'avant-bras et l'amplitude distale motrice du nerf tibial font exception à la similitude des différences G/D et T1/T2. En effet, dans ces cas, la variabilité temporelle est nettement inférieure à la variabilité G/D (Tableaux 1 et 2).

Les coefficients de variation entre les valeurs mesurées en $\mathrm{T} 1$ et en $\mathrm{T} 2$ sont pratiquement identiques aux valeurs moyennes des différences $\mathrm{T} 1 / \mathrm{T} 2$. Les coefficients de variation sont particulièrement faibles (nettement inférieurs à $10 \%$ ) pour les paramètres et nerfs suivants :

- la latence minimale de l'onde F de tous les nerfs étudiés ;

- l'amplitude distale motrice des nerfs ulnaire et tibial ;

- l'amplitude sensitive du nerf radial.

Pour ces différents nerfs et paramètres, les coefficients de corrélation entre les valeurs enregistrées en $\mathrm{T} 1$ et $\mathrm{T} 2$ sont supérieurs à 0,85 (Tableau 3).

L'étude de covariance révèle que les différences constatées entre les valeurs enregistrées en $\mathrm{T} 1$ et $\mathrm{T} 2$ ne sont pas, en règle générale, expliquées par des modifications significatives de l'intensité de stimulation entre les 2 examens. Trois exceptions doivent être mentionnées, à savoir la VC sensitive du nerf radial et l'amplitude des potentiels sensitifs des nerfs ulnaire et sural. En effet, les variations concernant ces paramètres et ces nerfs sont significativement et positivement corrélées $(p<0,05)$ aux variations d'intensité de stimulation entre les mesures effectuées en T1 et T2. La température est partiellement responsable des différences T1/T2 observées, en particulier pour les paramètres évaluant la $\mathrm{VC}$ nerveuse des segments terminaux et, dans une moindre mesure l'amplitude des réponses motrices et sensitives distales. En effet, les variations de latences distales motrices de l'ensemble des nerfs étudiés et les variations de VC sensitive (à l'exception du nerf ulnaire et du nerf cutané médial de l'avant-bras) sont significativement liées $(p<0,05)$ aux variations de température cutanée entre les explorations menées en T1 et T2. De la même manière, les variations de l'amplitude distale motrice $\mathrm{du}$ nerf tibial et les variations d'amplitude des potentiels sensitifs des nerfs sural et cutané médial de l'avant-bras sont significativement liées par une relation inverse $(p<0,05)$ avec les variations de température entre les examens réalisés en $\mathrm{T} 1$ et $\mathrm{T} 2$.

\subsection{Cas particulier de la persistance des ondes $F$ pour le nerf tibial}

La persistance de l'onde $\mathrm{F}$ lors de la stimulation du nerf tibial à la cheville est toujours de $100 \%$ dans notre population de 30 sujets sains et ce à gauche, à droite, en T1 et en T2. Les corrélations entre les valeurs enregistrées à gauche et à droite ou en $\mathrm{T} 1$ et $\mathrm{T} 2$ sont donc maximales $(r=1)$, tandis que les coefficients de variation et les limites de symétrie et de reproductibilité temporelle sont nuls $(=0)$ (Tableaux 1 et 3 ).

\section{Discussion}

Avant d'aborder la discussion des résultats de cette étude consacrée à la variabilité des données neurographiques, quelques précisions d'ordre méthodologique doivent être apportées. La taille du groupe de volontaires sains est modeste 
$(n=30)$ en comparaison à des travaux similaires rapportés dans la littérature où l'échantillon étudié est souvent supérieur à 100 sujets $[5,7-10,12,16,21]$ ou le nombre de répétitions d'une même mesure supérieur à 2 [1,2]. Pourtant, les variabilités, tant $\mathrm{G} / \mathrm{D}$ que temporelle, calculées dans notre travail sont souvent inférieures à celles mentionnées dans ces études. Deux explications permettent de rendre compte de ces différences. Ou bien le hasard a sélectionné un groupe de sujets sains particulièrement homogène sur le plan neurophysiologique, ou bien les choix méthodologiques permettent d'expliquer ces différences. Le choix d'une étude prospective avec sélection de volontaires sains permet une plus grande rigueur méthodologique par rapport à une analyse rétrospective de données enregistrées chez des patients [5]. Dans notre travail, contrairement à d'autres [5,16], toutes les données neurographiques sont recueillies par une seule et même personne sur le même électromyographe ; ce qui permet d'éliminer toute variabilité interexaminateur [11,21]. Nous avons également opté pour un placement automatique des curseurs de latence et d'amplitude sans possibilité de correction manuelle. La sélection de sujets jeunes (âge moyen de $22 \pm 2$ ans) permet de minimiser les lésions tronculaires ou radiculaires infracliniques. Enfin, nous avons souhaité nous placer dans des conditions proches d'un suivi neurophysiologique de patient souffrant d'une neuropathie périphérique ; ce qui nous a amené lors de l'évaluation à T2, à optimiser les valeurs relatives à la taille des réponses motrices et sensitives (cf. le chapitre matériel et méthodes). À l'inverse, d'autres choix ont probablement exagéré les variabilités enregistrées, en particulier temporelles. En effet, la réalisation de 2 tests à 3 mois d'intervalle expose à une plus grande fluctuation des mesures que si elles sont effectuées dans un délai plus court [15]. Et ce d'autant plus que nous avons pris l'option de ne pas corriger les données recueillies ou de réchauffer préalablement le membre étudié si la température cutanée était jugée basse. Ce parti pris se justifie par le souhait d'étudier l'influence de la température sur la variabilité temporelle. Par ailleurs, les corrections des données neurographiques en fonction de la température restent délicates en raison notamment de la non linéarité entre $\mathrm{VC}$ et température cutanée [12]. Quant au réchauffement des membres, il s'agit d'une manœuvre dont il est difficile de mesurer les effets puisqu'elle n'agit que de façon indirecte sur les structures étudiées. Néanmoins, il reste indéniable que la température est un facteur affectant significativement les données neurographiques, et donc les valeurs absolues mesurées et les différences G/D et temporelles calculées dans ce travail, en particulier pour les paramètres étudiant les segments distaux des membres (voir ci-dessous). Un autre point concerne la détermination de l'amplitude des réponses F. En effet, en choisissant une mesure pic à pic après superposition de l'ensemble des traces enregistrées, nous avons indéniablement privilégié l'aspect pratique et rapide de la mesure par rapport à sa signification physiologique. Concernant le protocole expérimental, il ne nous a pas semblé raisonnable d'étudier de façon exhaustive l'ensemble des paramètres neurographiques moteurs et sensitifs enregistrables aux membres supérieurs et inférieurs. Nous avons choisi de mettre en avant les paramètres pour lesquels nous attendions une grande variabilité (amplitudes des réponses motrices et sensitives et des ondes F, VC des segments courts, persistance des réponses F) ou ceux que nous jugions insuffisamment exploités dans les bilans neurophysiologiques de routine (ondes F). De ce fait, les VC motrices tronculaires des segments antébrachiaux et jambiers ont été quelque peu négligées. Cependant, concernant les VC, nos résultats indiquent que les variabilités calculées dépendent davantage de la longueur du segment que du nerf étudié (voir ci-dessous et Tableaux 1 et 2). Enfin, le choix d'une population de sujets jeunes amène inévitablement la question : les conclusions de cette étude sont-elles applicables à une population plus âgée qui aurait probablement été plus représentative de la tranche d'âge des patients habituellement examinés ? À notre connaissance, il n'existe pas d'étude évaluant le coefficient de variation des données neurographiques en fonction de l'âge. En revanche, la dispersion des données autour de la moyenne (appréciée par la déviation standard) en fonction de l'âge est facilement accessible pour la plupart des paramètres neurographiques. Dans notre expérience, fondée sur la constitution de normes propres à notre laboratoire, soit la valeur du paramètre étudié et sa dispersion autour de la moyenne augmentent en fonction de l'âge (latences distales motrices ou latences des ondes $\mathrm{F}$ ) ou bien le phénomène inverse est observé (amplitudes et VC). Cependant, lorsque la dispersion est exprimée en valeur pourcentuelle par rapport à la moyenne, elle est fréquemment identique chez les sujets jeunes ou âgés. Parfois, l'impression d'une dispersion plus grande des données à un des âges extrêmes est liée à une distribution de type exponentiel et la simple normalisation de cette distribution, en considérant le logarithme de la variable étudiée, permet de constater que la dispersion par rapport à la moyenne devient constante. Dès lors, la sous-estimation des limites de symétrie et de reproductibilité temporelle liée à l'étude d'une population très jeune n'est certainement pas systématique pour l'ensemble des variables étudiées. Néanmoins, une évaluation similaire dans une population plus âgée complèterait utilement notre étude.

Dans ce travail, d'une façon générale, lorsque l'on s'intéresse à la variabilité $\mathrm{G} / \mathrm{D}$ ou temporelle de la taille des réponses motrices et sensitives évoquées par des techniques neurographiques de surface, il n'y a pas d'intérêt particulier à considérer les valeurs de surface plutôt que les valeurs d'amplitude. En effet, les variabilités moyennes enregistrées pour ces 2 paramètres sont quasiment identiques. Dans le même ordre d'idée, la détermination de la latence moyenne ou maximale des ondes $\mathrm{F}$ ne permet pas de réduire la variabilité des mesures par rapport à l'utilisation de la latence minimale des réponses $\mathrm{F}$ qui, en outre sur le plan pratique, offre l'avantage supplémentaire d'être souvent plus facile à déterminer que les valeurs moyennes ou maximales (Tableaux 1 et 2).

Le coefficient de variation, outil statistique d'évaluation de la variabilité analytique, est quasiment systématiquement 
superposable aux valeurs moyennes de différences T1/T2 (Tableaux 1-3). Les différences constatées entre les 2 examens réalisés à 3 mois d'intervalle sont donc essentiellement expliquées par la variabilité analytique de la méthode utilisée. L'absence de fluctuation biologique des données neurographiques avait déjà été soulignée par Honet et al. [15]. Cette variabilité analytique résulte des modifications de la position des électrodes de surface, des erreurs de mesure des distances parcourues par les potentiels sensitifs et moteurs, des erreurs de positionnement des marqueurs de latence et d'amplitude et des modifications d'impédance cutanée et de température $[1,3,4,6,13,18,20,22]$. En effet, notre étude de covariance indique que les différences entre les résultats enregistrés à T1 et T2 sont en partie explicables par des modifications de la température cutanée, en particulier pour les paramètres évaluant la conduction nerveuse des segments distaux, à savoir la latence distale motrice de tous les nerfs étudiés et la VC sensitive de la plupart des nerfs explorés à l'exception des nerfs cutané médial de l'avant-bras et ulnaire. Bleasel et Tuck [2] avaient déjà mentionné le peu d'influence de la température sur la variabilité temporelle de la VC sensitive du nerf ulnaire. Ces mêmes auteurs avaient également montré que l'amplitude des réponses motrices et sensitives est moins sensible aux fluctuations de température que les paramètres évaluant la conduction nerveuse ; ce que nous confirmons dans le présent travail. En effet, l'étude de covariance ne montre une influence significative de la température sur les différences d'amplitudes mesurées en T1 et T2, que pour l'amplitude distale motrice du nerf tibial et l'amplitude des potentiels sensitifs des nerfs sural et cutané médial de l'avant-bras. Dès lors, si les paramètres et les nerfs précités sont utilisés à l'occasion d'un suivi neurophysiologique d'un patient souffrant d'une neuropathie périphérique, il est impératif de vérifier que les différences constatées entre 2 examens ne sont pas explicables par des variations significatives de la température cutanée. L'étude de covariance a également démontré que les différences constatées entre les examens réalisés en $\mathrm{T} 1$ et $\mathrm{T} 2$ pouvaient parfois s'expliquer par des variations d'intensité de stimulation, en particulier pour la VC sensitive du nerf radial et l'amplitude du potentiel sensitif des nerfs ulnaire et sural. Ce résultat souligne que les techniques neurographiques, en particulier les techniques de neurographie sensitive, restent techniquement délicates et que, même avec de l'expérience, il est parfois difficile d'éviter une stimulation infraliminaire, voire une stimulation trop intense.

En général, les différences G/D sont également proches ou identiques aux différences temporelles (Tableaux 1 et 2). Il semble donc que, globalement, les différences G/D traduisent également la variabilité analytique de la méthode utilisée. Honet et al. [15] arrivent aux mêmes conclusions dans leur étude consacrée à la variabilité des VC nerveuses. Dans le cas de l'amplitude sensitive des nerfs cutanés latéral et médial de l'avant-bras et de l'amplitude distale motrice du nerf tibial, les différences G/D sont beaucoup plus élevées que les différences T1/T2 (Tableaux 1 et 2). Dans ces cas particuliers, à la variabilité analytique, s'ajoute vraisemblablement une variabilité anatomique constitutionnelle significative.

Les résultats concernant la variabilité, tant G/D que temporelle, des paramètres évaluant la VC nerveuse confirment que la reproductibilité des mesures est d'autant meilleure que le segment nerveux étudié est long (Tableaux 1 et 2). Il se vérifie donc [16] que les courtes distances sont particulièrement adaptées à l'étude des troubles focaux de la conduction nerveuse en dépit d'une imprécision de mesure importante, tandis que les longues distances offrent une fiabilité de mesure élevée et doivent être privilégiées dans l'exploration et le suivi des processus avec démyélinisation diffuse ou multifocale. Cependant, dans certaines situations pathologiques, la détermination de la latence minimale de l'onde F peut être difficile à préciser en raison d'une très faible persistance (en particulier pour le nerf péronier), ou de la présence de réponses intermédiaires (réflexes d'axone ou autres). En pratique, ces situations restent relativement rares et ne doivent certainement pas remettre en cause l'utilité de ce paramètre.

\subsection{Différences gauche/droite et limites de symétrie}

Les valeurs normatives sont une donnée cruciale dans la pratique médicale et en particulier lors de l'application des techniques électroneuromyographiques. L'étude des différences G/D chez des sujets contrôles et l'établissement de limites de symétrie offrent l'avantage potentiel de pouvoir considérer le patient comme son propre témoin. Les données neurographiques ne sont plus alors dépendantes de l'âge, de la taille ou de la température (pour autant qu'elle soit symétrique) des patients examinés. Dès lors, en considérant les différences $\mathrm{G} / \mathrm{D}$, la dispersion des données de la population contrôle peut s'avérer plus faible que lorsque la valeur absolue du paramètre étudié est prise en compte. Lorsque la dispersion des données est moindre, les limites de normalité sont plus étroites et la sensibilité de la méthodologie à détecter une pathologie est accrue. Dans ce travail, nous constatons que les limites de symétrie G/D offrent une plus grande sensibilité que les limites établies sur base de la moyenne \pm 2 écart types, lorsque les corrélations entre les valeurs enregistrées à gauche et à droite sont élevées $(>0,75)$ et que les coefficients de variation, ou la variabilité temporelle moyenne, entre 2 examens réalisés successivement dans le temps sont bas (nettement inférieurs à $10 \%$ ). Ces 2 conditions sont toujours remplies pour la latence minimale de l'onde $\mathrm{F}$ de tous les nerfs étudiés. L'utilisation des limites de symétrie concernant ce paramètre est donc vraisemblablement un des meilleurs moyens d'apprécier une lésion unilatérale monotronculaire ou monoradiculaire, même si la zone de démyélinisation est localisée, comme c'est le cas dans un syndrome canalaire ou une compression radiculaire par une hernie discale.

Pour les autres paramètres appréciant la VC nerveuse, l'association d'un coefficient de corrélation G/D élevé et d'un faible coefficient de variation T1/T2 est remplie pour la VC sensitive des nerfs ulnaire, cutané latéral de l'avant-bras 
et radial, et pour la latence distale motrice des nerfs ulnaire et péronier. Pour ces différents nerfs et paramètres, la limite de symétrie G/D est de l'ordre de $15 \%$ lorsque l'on considère des segments courts $(8 \mathrm{~cm})$ et peut descendre jusqu'à 5-6\% lorsque l'on considère des segments plus longs, comme c'est le cas pour le nerf cutané latéral de l'avant-bras $(14 \mathrm{~cm})$ et radial sur le versant sensitif $(12 \mathrm{~cm})$.

Concernant le paramètre amplitude, les nerfs pour lesquels il semble particulièrement utile de considérer comme valeurs normatives les limites de symétrie G/D sont le nerf radial sensitif (limite de symétrie à $25 \%$ ) et sur le plan moteur, les nerfs ulnaire (limite de symétrie à $25 \%$ ) et tibial (limite de symétrie à $34 \%$ ). Pour les autres nerfs, les limites de symétrie peuvent dépasser les $50 \%$, voire atteindre la valeur de $69 \%$ pour l'amplitude du potentiel sensitif du nerf cutané médial de l'avant-bras. Dans ces circonstances, les limites de symétrie G/D n'offrent vraisemblablement pas une meilleure sensibilité que les valeurs normatives établies par la moyenne \pm 2 écart types.

Il existe une autre circonstance où l'utilisation des limites de symétrie G/D offre un intérêt potentiel, même si les corrélations G/D sont faibles et les coefficients de variation élevés. Il s'agit des situations dans lesquelles la dispersion des données au sein de la population de référence est telle que la valeur de 2 écart types est égale ou supérieure à la valeur moyenne du paramètre étudié. Dans ce cas, la limite inférieure de la normale est nulle ou négative, ce qui, sur le plan pratique, est inutilisable. Cette situation est rencontrée pour l'amplitude des réponses $\mathrm{F}$ de tous les nerfs étudiés et pour la persistance des réponses $\mathrm{F}$ du nerf péronier. Cette dispersion importante par rapport à la moyenne est vraisemblablement liée en partie à la multitude des afférences qui conditionnent l'excitabilité motoneuronale spinale et donc la production des ondes F. La limite de symétrie G/D pourcentuelle, établie par le percentile 95 , pour ces différents paramètres est globalement de l'ordre de $100 \%$ (Tableau 3).

\subsection{Différences T1/T2, limites de variabilité temporelle ou limites de stabilité}

Depuis que des traitements sont proposés dans le cadre de certaines neuropathies périphériques (dysimmunes, dysmétaboliques, voire paranéoplasiques), le neurophysiologiste est de plus en plus souvent sollicité pour réaliser des suivis longitudinaux de patients. Le but de ces suivis neurophysiologiques est de pouvoir affirmer si la neuropathie dont souffre un patient reste stable, s'améliore ou s'aggrave. Les limites de variabilité temporelle concernant les données neurographiques constituent donc des données essentielles pour évaluer l'évolution d'une neuropathie périphérique. En effet, le choix des nerfs et des paramètres à privilégier lors d'un suivi neurophysiologique doit prendre en compte 2 types d'information. Le premier choix doit se porter sur des nerfs qui sont intéressés par le processus pathologique dont on souhaite apprécier l'évolutivité. Le second choix doit prendre en considération le degré de variabilité analytique du paramètre étudié en fonction du nerf choisi. Ici aussi, les nerfs et les paramètres qu'il faut privilégier concernent ceux qui associent une corrélation T1/T2 élevée et des coefficients de variation faibles. Dans l'appréciation de l'évolution des VC nerveuses, c'est à nouveau la latence minimale des ondes $\mathrm{F}$ de l'ensemble des nerfs étudiés qui offre les meilleurs coefficients de corrélation et de variation. Sur le plan de l'amplitude des réponses motrices et sensitives, il semble qu'il faille privilégier l'amplitude distale motrice des nerfs ulnaire et tibial et l'amplitude du potentiel sensitif du nerf radial.

Les coefficients de variation et de corrélation concernant le paramètre amplitude des réponses $\mathrm{F}$ sont globalement assez médiocres. Néanmoins en pratique neurophysiologique de routine, l'utilisation de limites de variabilité temporelle (de l'ordre de $100 \%$ pour les différents nerfs moteurs, Tableau 3) reste utile, notamment pour apprécier la levée de blocs de conduction très proximaux dans le cadre des polyradiculonévrites aiguës, voire chroniques (Fig. 4).

La persistance des ondes $\mathrm{F}$ comme reflet indirect de la présence de blocs de conduction proximaux constitue en théorie un paramètre intéressant. Cependant, la relation inverse très étroite qui existe entre la persistance et la variabilité de la persistance semble introduire un biais rendant l'utilisation de ce paramètre peu utile en pratique (Fig. 3). En effet, lorsque la persistance moyenne est faible, comme c'est le cas pour le nerf péronier, la variabilité temporelle est élevée et les limites de stabilité sont très larges. En revanche, lorsque la persistance de l'onde F est élevée, la variabilité est faible y compris dans des conditions pathologiques. Finale-
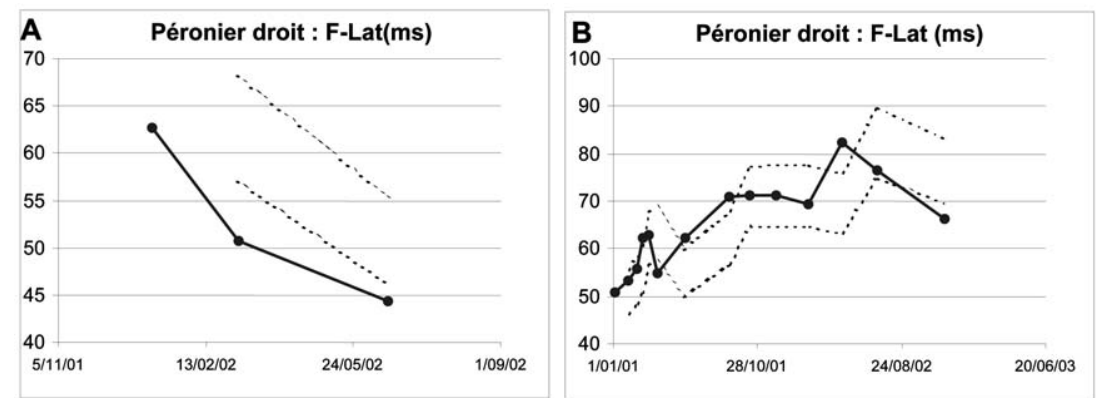

Fig. 4. Suivi neurophysiologique de la latence minimale de l'onde F (F-Lat) du nerf péronier chez un patient atteint d'un syndrome de Guillain Barré (A) et chez un autre souffrant d'une polyradiculonévrite chronique (B). Chaque disque noir représente un examen et les lignes discontinues indiquent les limites de variabilité temporelle ou de stabilité calculées lors de chaque exploration de contrôle sur la base de la valeur obtenue précédemment. 
ment, des fluctuations significatives de la persistance des ondes F ne s'observent que lors de la phase précoce de récupération des polyradiculonévrites aiguës telles que le syndrome de Guillain-Barré.

Enfin, une question qui reste partiellement non résolue est de savoir si les limites de variabilité temporelle établies dans une population contrôle peuvent être appliquées sans restriction à des patients présentant une neuropathie périphérique. Dans une étude consacrée également à la reproductibilité des données neurographiques, Kimura [16] répond en partie à cette question. En effet, dans ce travail, il étudie la reproductibilité de certaines données neurographiques à la fois dans un groupe de volontaires sains et dans un groupe de patients avec une neuropathie diabétique. Il s'avère que les variabilités dans les 2 groupes sont très équivalentes avec parfois une reproductibilité meilleure dans le groupe des patients, notamment concernant la VC sensitive et l'amplitude du potentiel sensitif du nerf médian. Seule la latence distale motrice du nerf tibial offre une moins bonne reproductibilité dans le groupe des patients avec une neuropathie diabétique que dans le groupe des sujets volontaires sains. Si donc, les limites de variabilité temporelle établies dans un groupe de sujets sains peuvent être extrapolées à des patients présentant une neuropathie périphérique, les suivis neurophysiologiques devraient se présenter tels qu'illustrés par la Fig. 4. Lors de chaque examen de contrôle, il est en effet possible d'établir, sur base des valeurs enregistrées lors de l'examen précédent, des limites de variabilité temporelle ou plus exactement des limites de stabilité au-delà desquelles il serait possible d'affirmer que le paramètre mesuré témoigne d'une aggravation ou d'une amélioration significative.

\section{Références}

[1] Bergmans J. On the variability of conduction velocity measurements on repeated examinations. Electromyography 1971;11:143-8.

[2] Bleasel AF, Tuck RR. Variability of repeated nerve conduction studies. Electro-encephalogr Clin Neurophysiol 1991;81:417-20.

[3] Bolton CF, Sawa GM, Carter K. The effects of temperature on human compound action potentials. J Neurol Neurosurg Psychiatry 1981;44: 407-13.

[4] Bromberg MB, Spiegelberg T. The influence of active electrode placement on CMAP amplitude. Electro-encephalogr Clin Neurophysiol 1997;105:385-9.
[5] Bromberg MB, Jaros L. Symmetry of normal motor and sensory nerve conduction measurements. Muscle Nerve 1998;21:498-503.

[6] Buchthal F, Rosenfalck A. Evoked action potentials and conduction velocity in human sensory nerves. Brain Res 1966;3:1-122.

[7] Buschbacher RM. Peroneal nerve F-wave latencies recorded from the extensor digitorum brevis. Am J Phys Med Rehabil 1999;78:S48-52.

[8] Buschbacher RM. Tibial nerve F-waves recorded from the abductor hallucis. Am J Phys Med Rehabil 1999;78:S43-7.

[9] Buschbacher RM. Ulnar nerve F-wave latencies recorded from the abductor digiti minimi. Am J Phys Med Rehabil 1999;78:S38-42.

[10] Buschbacher RM. Median nerve F-wave latencies recorded from the abductor pollicis brevis. Am J Phys Med Rehabil 1999;78:S32-7.

[11] Chaudhry V, Corse AM, Freimer ML, Glass JD, Mellits ED, Kuncl RW, et al. Inter- and intraexaminer reliability of nerve conduction measurements in patients with diabetic neuropathy. Neurology 1994;44:1459-62.

[12] Claus D, Mustafa C, Vogel W, Herz M, Neundorfer B. Assessment of diabetic neuropathy: definition of norm and discrimination of abnormal nerve function. Muscle Nerve 1993;16:757-68.

[13] Corbat F. Über Messungen der Leitgeschwindigkeit am peripheren Nerven und deren Verwertung in der Klinik. Dtsch Z Nervenheilk 1961;182:652-7.

[14] Ferrante MA, Wilbourn AJ. The utility of various sensory nerve conduction responses in assessing brachial plexopathies. Muscle Nerve 1995;18:879-89.

[15] Honet JC, Jebsen RH, Perrin EB. Variability of nerve conduction velocity determinations in normal persons. Arch Phys Med Rehabil 1968;49:650-4.

[16] Kimura J. Facts, fallacies, and fancies of nerve conduction studies: twenty-first annual Edward H. Lambert Lecture. Muscle Nerve 1997; 20:777-87.

[17] Lang AH, Puusa A. Dual influence of temperature on compound nerve action potential. J Neurol Sci 1981;51:81-8.

[18] Mayer RF. Nerve conduction studies in man. Neurology 1963;13: 1021-30.

[19] Olney RK. AAEM minimonograph \#38: neuropathies in connective tissue disease. Muscle Nerve 1992;15:531-42.

[20] Raynor EM, Preston DC, Logigian EL. Influence of surface recording electrode placement on nerve action potentials. Muscle Nerve 1997; 20:361-3.

[21] Salerno DF, Werner RA, Albers JW, Becker MP, Armstrong TJ, Franzblau A. Reliability of nerve conduction studies among active workers. Muscle Nerve 1999;22:1372-9.

[22] Trojaborg W. Motor nerve conduction velocities in normal subjects with particular reference to the conduction in proximal and distal segments of median and ulnar nerve. Electroencephalogr Clin Neurophysiol 1964;17:314-21.

[23] Valensi P, Attali JR, Gagant S. Reproducibility of parameters for assessment of diabetic neuropathy. The French Group for Research and Study of Diabetic Neuropathy. Diabet Med 1993;10:933-9. 